\title{
Analysis of melatonin content and gene expression in chinese cherry leaves
}

\author{
Xin Wang ${ }^{1}$. Tian Shen ${ }^{1}$, Xuefeng Zhang ${ }^{1}$, Peng Hu${ }^{1}$, Hongxia Tu ${ }^{1}$, Gui-hong Zhou ${ }^{2}$, Dong Liang ${ }^{1,3}$ and Hui Xia ${ }^{1,3, a}$ \\ ${ }^{1}$ Collega of Horticulture, Sichuan Agricultural University, Chengdu, Sichuan, China \\ ${ }^{2}$ Science Technology \& Agriculture Livestock Bureau of Wenchuan county, Aba Tibetan and Qiang Autonomous Prefecture, Sichuan \\ 623000, China \\ ${ }^{3}$ Insitute of pomology and Olericulture, Sichuan Agricultural University, Chengdu, Sichuan, China
}

\begin{abstract}
To investigate the accumulation characteristics and mechanism of melatonin in cherry leaves of different leaf ages, the melatonin content and the expression of synthetic genes in cherry leaves of different leaf ages were measured. The results showed that the highest melatonin content was found in young leaves of cherry. By analyzing the expression of genes for melatonin synthesis in leaves of different leaf ages, it was found that there was a good correlation between gene expression and melatonin content, and the trend was similar, both young leaves were higher than mature leaves and old leaves. By measuring the melatonin content in cherry leaves and the expression of genes for melatonin synthesis, this paper revealed the accumulation rule of melatonin in cherry leaves, and provided a reference for studying the characteristics of melatonin accumulation in plant leaves.
\end{abstract}

\section{Introduction}

Melatonin (N-acetyl-5-methoxytryptamine) is a newly discovered indoles hormone widely found in animals, plants and microorganisms. It is an amphiprotic molecules that can move in and out of cells freely and react with hydroxyl radicals and peroxides $[1,2]$. Studies on melatonin have found that it can effectively remove hydroxyl free radicals, peroxy free radicals, hydrogen peroxide, superoxide anion free radicals and singlet oxygen, and its antioxidant capacity is higher than that of carotenoids, glutathione, VC and VE [3]. There are few studies on the accumulation of melatonin in planting leaves.

In this study, the content of melatonin in leaves of different leaf ages of cherry was determined, and the expression of genes in melatonin synthesis was analyzed to investigate the relationship between melatonin accumulation and leaf ages of cherry leaves, as well as the gene expression rules of melatonin synthesis in leaves of different leaf ages.

\section{Materials and methods}

\subsection{Material preparation and handling}

\subsubsection{Collect leaves}

When the cherries are ripe, young leaves, mature leaves and old leaves were collected and put into an ice box. A total of 3 sets of leaves were collected, of which 60 pieces were collected as a group (10 pieces per set, 6 in total), and immediately sent to the laboratory for packaging, quick-frozen with liquid nitrogen, stored in the refrigerator at $-80^{\circ} \mathrm{C}$, the content of melatonin was determined, and RNA was extracted.

\subsection{Melatonin content determination}

High performance liquid chromatography-fluorescence assay was used. The $0.5 \mathrm{~g}$ leaves were ground into homogenate in $5 \mathrm{ml}$ methanol without light. Then it was extracted by $200 \mathrm{~W}$ ultrasonic oscillation for $30 \mathrm{~min}$ and centrifuged at $10000 \mathrm{r} / \mathrm{min}$ for $15 \mathrm{~min}$. The supernatant was taken $2 \mathrm{ml}$, filtered with $0.22 \mathrm{um}$ organic filter membrane for chromatographic analysis. The samples were separated on a nertsil ODS-3C18 column $(4.6 \times 250 \mathrm{~mm})$ with mobile phase (Water: methanol: acetic acid $=44.9: 55: 0.1$ ) at a flow rate of $0.8 \mathrm{ml} \mathrm{min}-1$. Melatonin was detected at $280 \mathrm{~nm}$ excitation and $384 \mathrm{~nm}$ emission wavelengths.

\subsection{Gene expression}

The expression patterns of five genes involved in melatonin synthesis, including TDC, T5H1, T5H2, SNAT and $A S M T$ were investigated by qRT-PCR. The primers were design using Primer3 based on our transcription data. Total RNA was extracted from samples according to the instructions of RNA prep pure Plant Kit. The concentration of total RNA samples was detected by nucleic acid protein analyzer, and the integrity was detected by $1.2 \%$ gel electrophoresis.

\footnotetext{
${ }^{\mathrm{a}}$ susanxia_2001@163.com
} 
According to the instructions of the Prime Script TM Reverse Transcriptase Kit, total RNA was used as a template for Reverse transcription into cDNA. After the mixing centrifugation, reverse transcription was performed on a PCR instrument $\left(37^{\circ} \mathrm{C}\right.$ for 15 minutes, $85^{\circ} \mathrm{C}$ for 5 seconds), then centrifugation was performed. The samples were diluted 10 times and the concentration of RT products was determined by Nnd-2000C nucleic acid protein detector. According to the results, all RT products were diluted to the same concentration for PCR amplification.

Table 1. Primers used in this study.

\begin{tabular}{cc}
\hline Primer & Squence \\
\hline \multirow{2}{*}{ PacTDC-real } & AACCGGGAAGATTTTTGCTT \\
& CACATGCCTCTCCTCAGTCA \\
PacT5H1-real & CAAAGCAATTCTGCAGGACA \\
& TTGGTTGAGGTCGCTCTCTT \\
PacT5H2-real & CAAATGCCACTCGACAGAGA \\
& ACCCTGGCACTACCAATCTG \\
PacSNAT-real & GATCAGAGGGGAATGACCAA \\
& GCCCTGATAACCAGGATCAA \\
PacASMT-real & GATGAGCCTAGCTCCAATGC \\
& GGCAAAGTCCCAAATCTCAA \\
Actin & CTTGCATCCCTCAGCACCTT \\
& TCCTGTGGACAATGGATGGA \\
\hline
\end{tabular}

Primers for the expression analysis of four genes were designed based on the sequences of the genes cloned by the research group. By reverse transcription of cDNA template, reference SYBR@ Premix Ex Taq ${ }^{\mathrm{TM}} \mathrm{II}$ kit instructions operate (reaction system for SYBR Premix Ex $\mathrm{Taq}^{\mathrm{TM}} 10.0 \mu \mathrm{L}$, primers respectively $0.4 \mu \mathrm{L}$, ddH2O $8.2 \mu \mathrm{L}$ ). PCR amplification was performed using BIO-RAD iQ5 ${ }^{\mathrm{TM}}$ real-time quantitative PCR instrument. The reaction procedure was: pre-denaturation at $95^{\circ} \mathrm{C}$ for $10 \mathrm{~min}$; Denatured at $95^{\circ} \mathrm{C}$ for $10 \mathrm{~s}$; Annealing at $60^{\circ} \mathrm{C}$ for $31 \mathrm{~s}$ and $72^{\circ} \mathrm{C}$ extension $20 \mathrm{~s}$, a total of 40 cycles. IQ5 ${ }^{\mathrm{TM}}$ real-time PCR Detection System was used to analyze gene expression data.

\subsection{Data processing and analysis}

Test data were processed and analyzed by Excel 2016.

\section{Results and analysis}

\subsection{Changes of melatonin content in leaves at different leaf ages}

The melatonin (MT) content in leaves of chinese cherry at different leaf ages was compared (figure 1). The melatonin content was as follows: young leaves $>$ mature leaves $>$ old leaves. The content of melatonin in young leaves was about $2.48 \mathrm{ug} / \mathrm{g}$, twice that in mature leaves and 3.6 times that in older leaves.

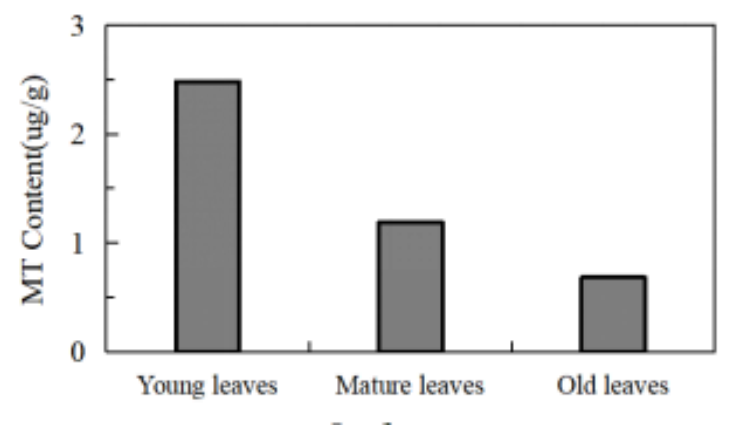

Figure 1. The content of melatonin in cherry leaves with different leaf ages.

\subsection{Gene expression profiles}

The expression levels of genes for melatonin synthesis in cherry leaves of different leaf ages were determined. The results showed that the expression level of the five genes was low in the leaves, and the expression level was highest in the young leaves, followed by that in the old leaves, and lowest in the mature leaves. The expression of PacT5H1 in leaves of different leaf ages showed the greatest difference, the expression of young leaves was 39 times that in mature leaves, and the expression of PacT5H2 showed the smallest difference. In young leaves, PacT5H1 expressed the most. Among mature leaves and old leaves, PacSNAT expressed the most. 

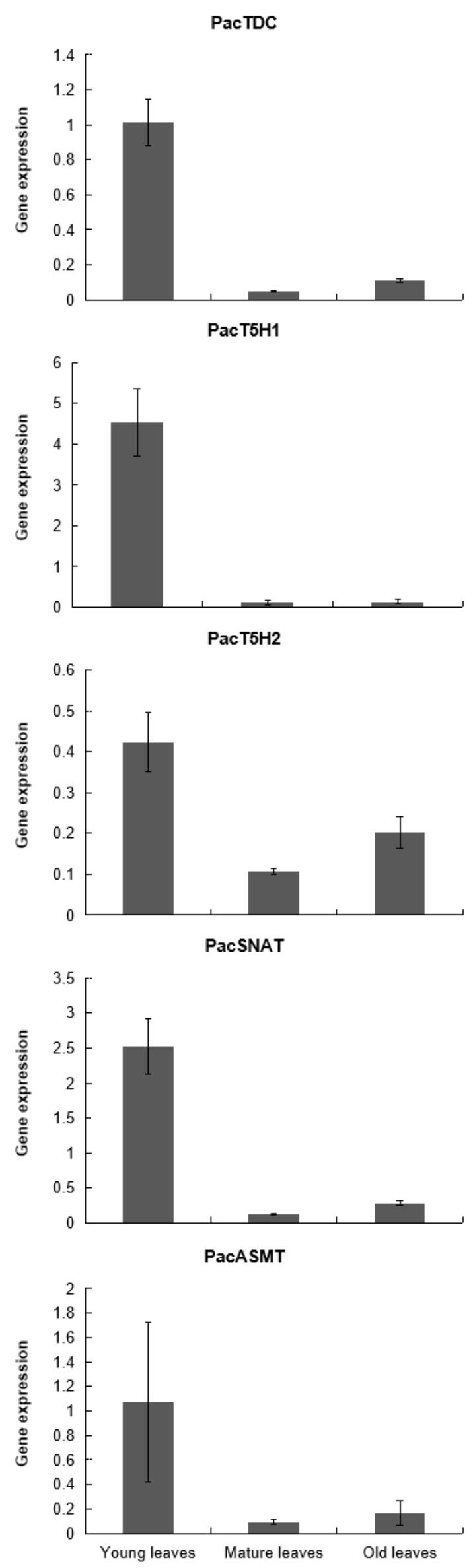

Figure 2. The expression pattern of melatonin synthesis genes.

\section{Conclusion}

The content of melatonin in leaves of different leaf ages is different, and the difference is large, mainly manifested as: young leaves $>$ mature leaves $>$ old leaves. Studies have found that melatonin levels in the mulberry leaves are characterized by: young leaves $>$ mature leaves $>$ old leaves [4], this is consistent with the test results, it shows that the accumulation pattern of melatonin in the blade is likely to be young leaves > mature leaves $>$ old leaves, predecessors also think melatonin is a kind of juvenile or aging antagonist material [5]. The experimental material is only for chinese cherry leaves, and the peculiar accumulation rule of melatonin at different leaf ages needs to be studied. In the analysis of the expression of genes for melatonin synthesis in leaves of different ages of chinese cherries, it was found that the expression of five genes in leaves was consistent with the change trend of melatonin content, all of which were young leaves $>$ mature leaves and old leaves. We found that although melatonin content was lower in old leaves than in mature leaves. The melatonin-related gene expression was higher in old leaves. In addition, in the current studies, the expression of PacTDC gene was prior to the synthesis of melatonin [6], both in fruit development and 24-hour rhythm studies. Although the expression level of PacTDC gene was not the highest in this experiment, it also played an important role in the synthesis of melatonin. It was found that the expression level of PacT5H1 was the highest in young leaves, and the expression level of PacSNAT was the highest in mature leaves and old leaves. Therefore, it is speculated that the expression of PacT5H1 plays a greater role in the synthesis of melatonin during the growth period of leaves. After the ripening of leaves, the expression levels of melatonin synthesis related genes were significantly decreased, and the expression of PacSNAT played a more significant role in melatonin synthesis. With the aging of mature leaves, in order to delay the aging of leaves, the expression level of genes related to melatonin synthesis increased slightly, but was still lower than that in young leaves, and the expression level of PacSNAT was still the highest. Studies have found that melatonin's effects in plants include protecting young tissues and delaying plant senescence [5]. Therefore, it can be believed that the main purpose of plant melatonin synthesis in young leaves is to avoid oxidative damage and protect young tissues, during which a relatively large amount of melatonin is synthesized. In the old leaves, the enzyme activity gradually decreased with the senescence of the leaves, and the gene expression level should be lower than that of the mature leaves and the young leaves. However, in order to delay senescence, the expression level of the gene for melatonin synthesis began to increase, which was higher than that of the mature leaves. However, at the same time, melatonin content was lower than that of mature leaves due to the decreased enzyme activity. This study provides a certain reference for the study of melatonin accumulation in plants, especially cherry leaves, and provides a new direction for the study of the expression of genes for melatonin synthesis. 


\section{Acknowledgement}

This work was financially supported by the Sichuan Science and Technology Project under Grant 2018JY0461 and Sichuan Innovation Training Program for College Students under Grant S201910626108.

\section{References}

1. C.S. Shida, A.M.L. Castrucci, M.T. Lamy-Freund. High melatonin solubility in aqueous medium. J. Journal of Pineal Research, 16 (4): 198-201, (1994)

2. L. Ceraulo, M. Ferrugia, L. Tesoriere, jr. Interactions of melatonin with membrane models: portioning of melatonin in AOT and lecithin reversed micelles. J. Journal of Pineal Research, 26 (2): 108-112, (1999)

3. T. DunXian, R. Hardeland, L.C. Manchester, jr. Functional roles of melatonin in plants, and perspectives in nutritional and agricultural science. J. Journal of Experimental Botany, 63 (2): 577-597, (2012)

4. P. Pothinuch, S. Tongchitpakdee. Melatonin contents in mulberry (Morus spp) leaves: Effects of sample preparation, cultivar, leaf age and tea processing. J. Food Chemistry, 128 (2): 415-419, (2011)

5. G. Biao, S. QingHua. Research progress on melatonin in horticultural crops. J. Chinese academy of agricultural sciences, 50 (12): 2326-2337, (2017)

6. Z. Yu, T. DunXian, L. Qiong, jr. Melatonin and its potential biological functions in the fruits of sweet cherry. J. Journal of Pineal Research, 55 (1): 79-88, (2012) 